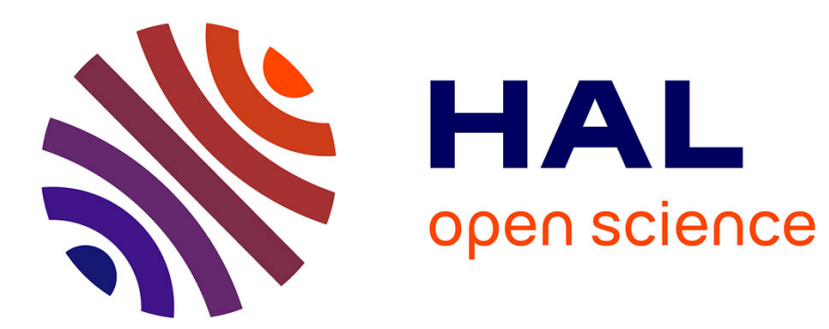

\title{
The FI-SDW formation in (TMTSF)2ClO4 investigated in the infrared
}

A. Gerrits, T. Janssen, J. Brooks, A. Wittlin, J. Perenboom, P. van Bentum

\section{To cite this version:}

A. Gerrits, T. Janssen, J. Brooks, A. Wittlin, J. Perenboom, et al.. The FI-SDW formation in (TMTSF)2ClO4 investigated in the infrared. Journal de Physique IV Proceedings, 1993, 03 (C2), pp.C2-299-C2-302. 10.1051/jp4:1993260 . jpa-00251343

\section{HAL Id: jpa-00251343 https://hal.science/jpa-00251343}

Submitted on 1 Jan 1993

HAL is a multi-disciplinary open access archive for the deposit and dissemination of scientific research documents, whether they are published or not. The documents may come from teaching and research institutions in France or abroad, or from public or private research centers.
L'archive ouverte pluridisciplinaire HAL, est destinée au dépôt et à la diffusion de documents scientifiques de niveau recherche, publiés ou non, émanant des établissements d'enseignement et de recherche français ou étrangers, des laboratoires publics ou privés. 


\title{
The FI-SDW formation in (TMTSF) ${ }_{2} \mathrm{ClO}_{4}$ investigated in the infrared
}

\author{
A.M. GERRITS, T.J.B.M. JANSSEN, J.S. BROOKS*, A. WITTLIN, J.A.A.J. PERENBOOM and \\ P.J.M. VAN BENTUM
}

High Field Magnet Laboratory and Research Institute for Materials, University of Nijmegen, NL-6525 ED Nijmegen, The Netherlands

${ }^{*}$ Department of Physics, Boston University and F.B. National Magnet Laboratory, M.I.T., Cambridge, Massachusetts 02139, U.S.A.

\begin{abstract}
From a magneto-optical study on (TMTSF) ${ }_{2} \mathrm{ClO}_{4}$ we determine the SDW gap as a function of magnetic field and temperature. The transition temperatures found for the resolved FI-SDW subphases mimic the established phase diagram. Furthermore, the data confirm the existence of a collective excitation of the SDW condensate at relatively high frequency, which implies that this mode is pinned rather strongly to the lattice. In additon, we observed an interesting relaxation effect near the $\mathrm{N}=0$ phase transition.
\end{abstract}

\section{Introduction}

The observation of the unusual field induced transition to a SDW state in the Bechgaard salts contributed highly to the present interest in these organic compounds. In Ref. 1 we reported far infrared measurements of the excitation spectrum at $0.4 \mathrm{~K}$, showing that in the FI-SDW subphases of (TMTSF) ${ }_{2} \mathrm{ClO}_{4}$ part of the charge carriers condense in a collective state with a clear gap near the Fermi level. The magnitude of the gap was the same in all sub-phases and independent of the value of the magnetic field in contrast to the predictions of the "standard model" [2]. At the phase boundaries the free carrier density changed discontinuously indicating a first order phase transition. This indicates that the gap is not uniform in $\mathbf{k}$ space, and that in all subphases there is a remaining free carrier density, related to the ungapped area of the Fermi surface. This free carrier density changes abruptly at the various phase transitions.

In addition, we reported observations of a collective mode excitation inside the gap and a pronounced coupling of the phonons with the SDW formation. In the present study we investigated the influence of temperature on the excitation spectrum of (TMTSF) ${ }_{2} \mathrm{ClO}_{4}$ between 2 and $45 \mathrm{~cm}^{-1}$ in fields up to $20 \mathrm{~T}$.

\section{The FI-SDW state studied with far infrared spectroscopy}

The single crystal samples were aligned in a gridlike structure and cooled as reported elsewhere [1] and mounted in the bore of either a $18 \mathrm{~T}$ superconducting magnet or a $20 \mathrm{~T}$ Bitter magnet. The magnetic field was applied along the c-axis, perpendicular to the grid. We used various FIR setups: an optically pumped molecular FIR laser, a microwave harmonic generator and a Grubb Parsons fourier spectrometer. For this grid geometry, the transmitted power in the spectral region of interest is polarized perpendicular to the grid and mainly depends on the conductivity along the b-axis. After the measurements we evaporated a gold film on the sample grid and measured it again for normalization purposes.

Figure 1 shows the FIR transmission through the grid as a function of the applied magnetic field at various temperatures. The transmission $\mathrm{T}$ is normalised on the transmission in zero field. A FIR energy of $8.3 \mathrm{~cm}^{-1}$ was used which corresponds to an energy well within the SDW gap. With increasing temperature we find the resolved FI-SDW phase transitions to decrease in number and to shift to higher fields. At low temperatures the transitions occur almost discontinuously, indicative of a first order nature of the phase transitions. At higher temperatures the transitions are broadened and the change in the FIR transmission becomes smaller, probably related to the thermally excited carriers above the SDW gap. 


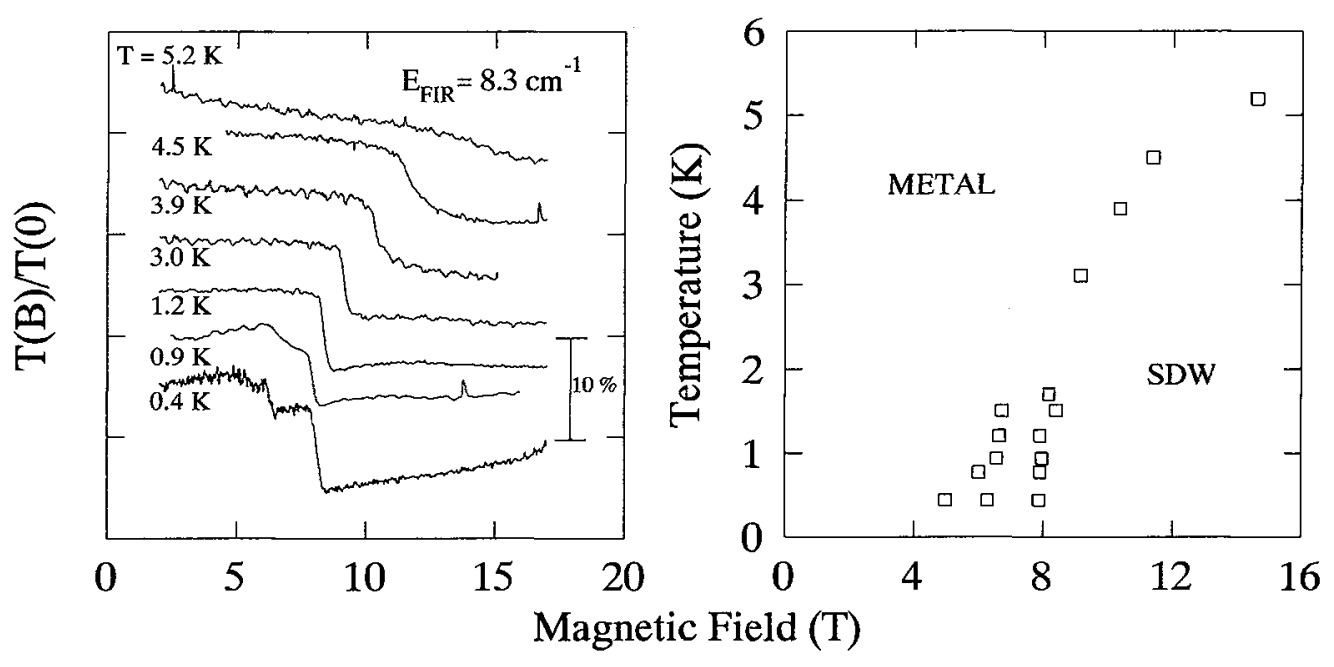

Figure 1: left: FIR transmission well within the SDW gap as a function of the applied magnetic field at different temperatures. The traces are offset for clarity. right: Deduced phase diagram.

Taking the midpoint of the steps in the transmission signal as the transition field we obtain the phase diagram drawn on the right side of figure 1. The agreement with the established phase diagram (see e.g. Ref. 3) is quite good, giving confidence that we are indeed optically probing the SDW formation.

In figure 2 we show the energy spectrum at $10 \mathrm{~T}$ for various temperatures. At this field we only probe the $\mathrm{N}=0$ phase. At low temperatures we observe a gaplike structure below $14 \mathrm{~cm}^{-1}$ as reported before [1]. With increasing temperature the gap edge does not shift to lower energies but remains at a fixed position. The signal strength inside the gap is nearly constant below the transition temperature. Both effects are quite different from a standard BCS-like mean field prediction.

At low frequencies we find a strong excitation mode which slightly hardens when going to lower temperatures. This confirms the earlier observations of this mode [1]. It is clear that the intensity of this mode is weakly temperature dependent until it vanishes near the critical temperature. This is in agreement with the assignment to a collective mode excitation of the SDW state, where the hardening at low temperatures is caused by a more effective pinning of the condensate.

In the region of the $17 \mathrm{~cm}^{-1}$ and the $32 \mathrm{~cm}^{-1}$ phonons, which are probably both related to zone folding due to the anion ordering superstructure at low temperatures, we find a strong change in the phonon lineshape when lowering the temperature into the SDW state. When the spectra are normalized to spectra taken on the gold-covered sample (see Fig. 3) it is indeed clear that the $17 \mathrm{~cm}^{-1}$ phonon peak decreases in intensity and becomes strongly asymmetric. This could be due to the increased coupling to electronic excitations just above the SDW gap. A similar effect occurs for the $32 \mathrm{~cm}^{-1}$ mode. Although unexpected for SDW systems, the changes in themselves are not very surprising given the additional incommensurate modulation which breaks translation symmetry and changes the selection rules for optically active $(k=0)$ phonon modes. However, it must be noted that both phonons are also present in the metallic state and the major effect of the SDW formation is a change in lineshape.

The fact that, above $T_{c}$, shallow remnants of the gap structure remain visible indicates a possible presence of SDW fluctuations with no long range magnetic order. It is interesting to note that similar effects occur in high $\mathrm{T}_{\mathrm{c}}$ superconductors.

In order to check the assignment of the $14 \mathrm{~cm}^{-1}$ edge to the SDW gap we performed FIR measurements on a similar grid of (TMTSF) ${ }_{2} \mathrm{PF}_{6}$ crystals, which also show a SDW transition however not field induced. Below the SDW ordering temperature which is $12 \mathrm{~K}$ at ambient pressure for this material, a decrease of the transmission signal below $32 \mathrm{~cm}^{-1}$ is observed. Near this edge we find no visible phonon structure in the spectra, and there are no corresponding features due to zone folding because there is no anion ordering in the $\mathrm{PF}_{6}$ compound. We find that the size of this gap is about twice as large as the sharp edge in the 


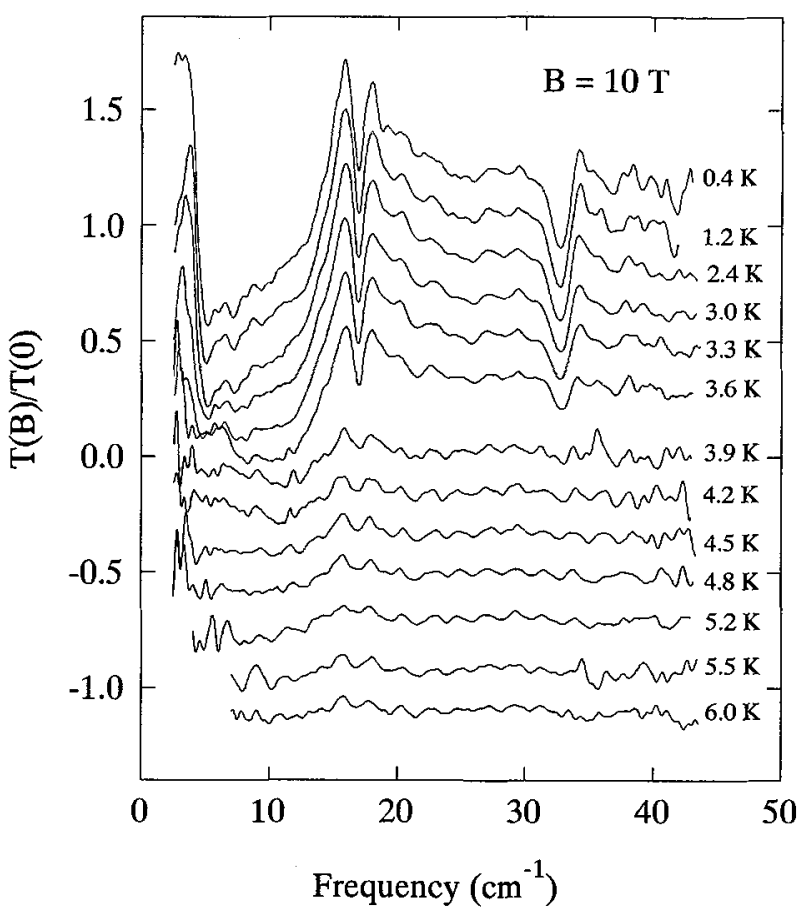

Figure 2: Normalized transmission, $\mathrm{T}(\mathrm{B}) / \mathrm{T}(0)$, as a function of frequency and temperature. The spectra are offset for clarity.

(TMTSF) $)_{2} \mathrm{ClO}_{4}$ spectra, in accordance with the twice as large value of $T_{\mathrm{c}}$ in (TMTSF) ${ }_{2} \mathrm{PF}_{6}$.

Finally, we present data which show an interesting memory effect observed for frequencies inside the gap. In figure $4 \mathrm{a}$, a fieldsweep of the normalized transmission signal $T(B) / T(0)$ at $7.2 \mathrm{~cm}^{-1}$ is depicted. When the field was swept up, we found the signal to show the usual steps at the FI-SDW transition fields. Just after the $N=0 \mathrm{SDW}$ state was entered at $8.0 \mathrm{~T}$, the field value was fixed. The signal relaxed very slowly (on the time scale of minutes) to the level corresponding to the previous $\mathrm{N}=1$ subphase. When after about 20 minutes the field was swept down, the signal was found to drop again to the level of the $\mathrm{N}=0$ state. At even lower fields the transmission rose again and after this, the usual steps of the phase transitions were observed. The long time scale for the relaxation shown in figure $4 \mathrm{~b}$ suggests either the presence of domain formation or a posible coupling to the nuclear spins of the organic molecules. The fact that the system relaxes back to the $\mathrm{N}=1$ state after it already made the transition to the $\mathrm{N}=0$ state rules out hysteresis effects or heating. The nucleation of domain structures also seems unlikely because the system already made the full phase transition. Also on the down sweep, the transition occurs on a much shorter timescale. The appearance of a memory effect while sweeping down in field suggests the build up of an internal magnetic field. A similar sort of effect was first predicted by Overhanser [5] for metallic systems. He suggested that an electronic system saturated via ESR can effectively polarize the nuclei by means of hyperfine interaction between electrons and nuclei. In the present system one can think of a similar effect where the electronic system carries a stationary magnetic moment which varies in space. The hyperfine interaction could effectively induce a coupled magnetization of the nuclei (presumably Se or $\mathrm{H}$ ) with the same periodicity as the electronic SDW. Azevedo et al. [6] estimated the local field, in (TMTSF) ${ }_{2} \mathrm{ClO}_{4}$ via NMR experiments, to be larger than $0.1 \mathrm{~T}$ on the Selenium sites. A very slow (minutes) proton relaxation rate was observed in the FI-SDW states and indications were found for a change in relaxation mechanism at the phase transition.

Because the FI-SDW formation is the result of a delicate energy balance it is possible that the lowest energy state for a given external field in the presence of the polarized nuclei is different from the state without nuclear polarization. This kind of magnetic coupling could also be the origin of the frequently observed 


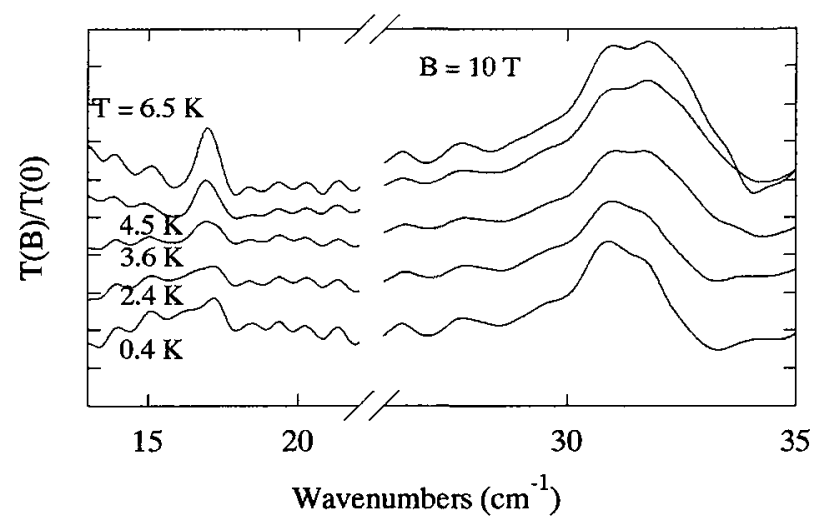

Figure 3: Transmission normalised to the transmission of the gold-covered sample near $17 \mathrm{~cm}^{-1}$ and $32 \mathrm{~cm}^{-1}$ at various temperatures. The spectra are offset for clarity.

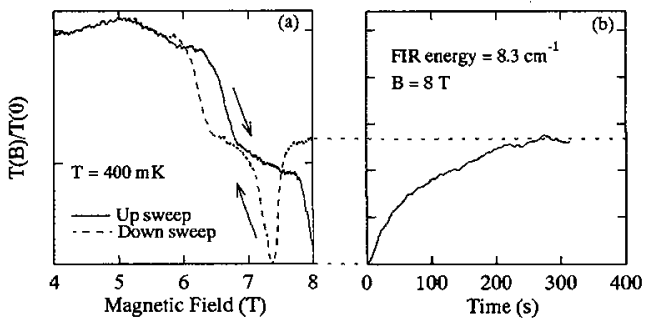

Figure 4: (a) Transmission as a function of magnetic field using a microwave frequency of $7.2 \mathrm{~cm}^{-1}$ (b) The relaxation of the transmission signal as a function of time at a fixed field of $8 \mathrm{~T}$. (see text)

hysteresis effects near the SDW transitions. It could also explain the unusual pinning of the SDW electronic groundstate to the lattice.

\section{Conclusion}

In conclusion, we present new spectroscopic data on the temperature dependence of the FI-SDW formation in (TMTSF) $)_{2} \mathrm{ClO}_{4}$. The value of the SDW gap is relatively insensitive to temperature. The temperature transition at high fields as well as the field transitions at low temperatures are of first order. The optically detected phase transitions follow closely the phase diagram inferred from thermodynamic measurements. Above $T_{\mathrm{c}}$ some shallow gap structure in the spectra remains. This indicates possible fluctuations of the FI-SDW state. We confirm the presence of a collective mode near $2.4 \mathrm{~cm}^{-1}$ which is weakly temperature dependent, indicating possible depinning at higher temperatures.

We report the first measurements of peculiar memory effects near the SDW subphase transitions, possibly indicating a magnetic coupling to the lattice.

We thank Paul Chaikin and coworkers at Princeton University for providing the samples.

\section{References}

[1] T.J.B.M. Janssen, A.S. Perel, A.M. Gerrits, W. Kang, J.S. Brooks, A. Wittlin, J.A.A.J. Perenboom, and P.J.M. van Bentum, Phys. Rev. B 46, (1992) 8663.

[2] G. Montambaux, and D. PoilBlanc, Phys. Rev. B 37, (1988) 1913.

[3] N.A. Fortune, J.S. Brooks, M.I. Graf, G. Montambaux, L.Y. Chaing, J.A.A.J. Perenboam, and D. Althof, Phys. Rev. Lett. 64, (1988) 621.

[4] M.J. Naughton, J.S. Brooks, L.Y. Chaing, R.V. Chamberlin, and P.M. Chaikin, Phys. Rev. Lett. 55, (1985) 969.

[5] A.W. Overhauser, Phys. Rev. Lett. 2, (1953) 411.

[6] L.J. Azevedo, J.M. Williams, and S.J. Compton, Phys. Rev. B 28, (1983) 6600. 